\title{
Probing nucleon strange and charm distributions with lattice QCD
}

\author{
Rui Zhang®, ${ }^{1,2}$ Huey-Wen Lin, ${ }^{1,2 *}$ and Boram Yoon $^{3}$ \\ ${ }^{1}$ Department of Physics and Astronomy, Michigan State University, East Lansing, Michigan 48824, USA \\ ${ }^{2}$ Department of Computational Mathematics, Science and Engineering, Michigan State University, \\ East Lansing, Michigan 48824, USA \\ ${ }^{3}$ Computer, Computational, and Statistical Sciences CCS-7, Los Alamos National Laboratory, \\ Los Alamos, New Mexico 87545, USA
}

(Received 3 July 2020; revised 15 December 2020; accepted 15 September 2021; published 18 November 2021)

\begin{abstract}
We present the first lattice-QCD calculation of the unpolarized strange and charm parton distribution functions using large-momentum effective theory. We use a lattice ensemble with $(2+1+1)$ flavors of highly improved staggered quarks generated by the MILC Collaboration, with lattice spacing $a \approx 0.12 \mathrm{fm}$ and $M_{\pi} \approx 310 \mathrm{MeV}$, and clover valence fermions with two valence pion masses: 310 and $690 \mathrm{MeV}$. We use momentum-smeared sources to improve the signal up to nucleon boost momentum $P_{z}=2.15 \mathrm{GeV}$, and determine nonperturbative renormalization factors in regularization independent momentum subtraction scheme. We compare our lattice results with the matrix elements obtained from matching the parton distribution functions (PDFs) from CT18NNLO and NNPDF3.1NNLO global fits. Our data support the assumptions of strange-antistrange and charm-anticharm symmetry that are commonly used in global PDF fits.

DOI: 10.1103/PhysRevD.104.094511
\end{abstract}

\section{INTRODUCTION}

Parton distribution functions (PDFs) provide a universal description of hadronic constituents as well as critical inputs for the discovery of the Higgs boson found at the Large Hadron Collider (LHC) through proton-proton collisions [1,2]. While the world waits for the next phase of LHC discovery focused on searching for new physics signatures, improvements in the precision with the known Standard-Model backgrounds will be crucial to discern these signals. For example, our knowledge of many Higgsproduction cross sections remains dominated by PDF uncertainties. Among the known PDFs, the strange and charm PDFs have particularly large uncertainties despite decades of experimental effort. In addition to their applications to the energy frontier, PDFs also reveal a nontrivial structure inside the nucleon, such as its momentum and spin distributions. Many ongoing and planned experiments at facilities around the world, such as the Brookhaven National Laboratory and the Jefferson Laboratory in the USA, GSI in Germany, J-PARC in Japan, or a future

\footnotetext{
*hwlin@pa.msu.edu
}

Published by the American Physical Society under the terms of the Creative Commons Attribution 4.0 International license. Further distribution of this work must maintain attribution to the author(s) and the published article's title, journal citation, and DOI. Funded by SCOAP . electron-ion collider (EIC), are set to explore the lessknown kinematics of nucleon structure and more.

In order to distinguish the flavor content (strange or charm) of the PDFs, experiments use nuclear data, such as neutrino scattering off heavy nuclei, and the current understanding of medium corrections in these cases is limited. Thus, the uncertainty in the strange PDFs remains large. In many cases, the assumptions $\bar{s}(x)=s(x)$ and $\bar{c}(x)=c(x)$ that are often made in global analyses can agree with the data merely due to the large uncertainty. At the LHC, strangeness can be extracted through the $W+c$ associatedproduction channel, but their results are rather puzzling. For example, ATLAS got the ratios of averaged strange and antistrange to the antidown distribution, $(s+\bar{s}) /(2 \bar{d})$, to be $0.96_{-0.30}^{+0.26}$ at $Q^{2}=1.9 \mathrm{GeV}^{2}$ and $x=0.023$ [3]. CMS performed a global analysis with deep-inelastic scattering data and the muon-charge asymmetry in $W$ production at the LHC to extract the ratios of the total integral of strange and antistrange to the sum of the antiup and antidown, at $Q^{2}=20 \mathrm{GeV}^{2}$, finding it to be $0.52_{-0.15}^{+0.18}$ [4]. Future highluminosity studies may help to improve our knowledge of the strangeness. In the case of the charm PDFs, there has been a long debate concerning the size of the "intrinsic" charm contribution, as first raised in 1980 [5] and developed in subsequent papers [6-8]..$^{1}$ It is important to distinguish this contribution from radiative contributions

\footnotetext{
${ }^{1}$ We refer interested readers to Ref. [9] and references within $[10,11]$ for a review of intrinsic-charm discussions.
} 
in future NNLO and N3LO global PDF analyses. $c(x)-$ $\bar{c}(x)$ provides an important check of the intrinsic charm contribution to the proton. Again, the current experimental data are too inconclusive to discriminate between various proposed QCD models, and future experiments at LHC or EIC could provide useful information in settling this mystery.

Although there exists a variety of model approaches to treat the structure functions, a nonperturbative approach from first principles, such as lattice QCD (LQCD), provides hope to resolve many of the outstanding theoretical disagreements and provides information in regions that are unknown or difficult to observe in experiments. In this work, we will be using the large-momentum effective theory (LaMET) framework $[12,13]$ to provide information on the Bjorken- $x$ dependence of the strange and charm PDFs. In the LaMET (or "quasi-PDF") approach, timeindependent spatially displaced matrix elements that can be connected to PDFs are computed at finite hadron momentum $P_{z}$. A convenient choice for leading-twist PDFs is to take the hadron momentum and quark-antiquark separation to be along the $z$ direction. On the lattice, we then calculate hadronic matrix elements

$$
h\left(z, P_{z}\right)=\langle P|\bar{\psi}(z) \Gamma W(z, 0) \psi(0)| P\rangle,
$$

where $\psi$ is the quark field (charm and strange in this calculation), $|P\rangle$ is the nucleon state in our case, $W(z, 0)$ is the spacelike Wilson-line product $\left(\prod_{n} U_{z}(n \hat{z})\right)$ with $U_{z}$ a discrete gauge link in the $z$ direction. There are multiple choices of operator in this framework that will recover the same light-cone PDFs when the large-momentum limit is taken; in this work, we will use $\Gamma=\gamma^{t}$ for unpolarized distribution, as suggested in Refs. [14-17]. The "quasi-PDF" $\tilde{q}\left(x, P_{z}\right)$ is then obtained from a Fourier transformation of the continuum-limit renormalized matrix elements $h^{R}$

$$
\tilde{q}\left(x, P_{z}\right)=\int \frac{d z}{4 \pi} e^{i x P_{z} z} h^{R}\left(z, P_{z}\right),
$$

where $x$ is the fraction of momentum carried by the parton relative to the hadron. For this first study of these quantities, we will neglect the lattice-spacing and finite-volume dependence. The quasi-PDF is related to the light-cone PDF at scale $\mu$ in $\overline{\mathrm{MS}}$ scheme through a factorization theorem

$$
\begin{aligned}
& \tilde{q}_{\psi}\left(x, P_{z}, \mu^{\overline{\mathrm{MS}}}, \mu^{\mathrm{RI}}, p_{z}^{\mathrm{RI}}\right) \\
& \quad=\int_{-1}^{1} \frac{d y}{|y|} C\left(\frac{x}{y},\left(\frac{\mu^{\mathrm{RI}}}{p_{z}^{\mathrm{RI}}}\right)^{2}, \frac{y P_{z}}{\mu^{\mathrm{MS}}}, \frac{y P_{z}}{p_{z}^{\mathrm{RI}}}\right) q_{\psi}\left(y, \mu^{\overline{\mathrm{MS}}}\right)+\cdots,
\end{aligned}
$$

where $p_{z}^{\mathrm{RI}}$ and $\mu^{\mathrm{RI}}$ are the momentum of the off-shell strange quark and the renormalization scale in the regularization independent momentum subtraction scheme (RI/MOM) nonperturbative renormalization (NPR), and $C$ is the perturbative matching kernel used in our previous works [18-21]. The residual terms, $\mathcal{O}\left(\frac{\Lambda_{\mathrm{OCD}}^{2}}{x^{2} P_{2}^{2}}, \frac{m_{N}^{2}}{P_{2}^{2}}\right)$, come from the nucleon-mass correction and higher-twist effects, suppressed by the nucleon momentum. In recent years, although there have been multiple PDF calculations using this approach [18-35] or alternative approaches, such as the pseudo-PDF [17,36-45] and the "good lattice cross sections" [46-50], only the "connected" contribution of the PDFs has been studied on the lattice so far. $^{2}$ We refer readers to a recent review article [52] for the most complete summary of the latest $x$-dependent LaMET-related calculations. This work is the first exploratory study to take on the challenges of the notorious "disconnected" contribution, an important next step toward flavor-dependent PDFs from lattice QCD.

\section{LATTICE SETUP}

We calculate the observables on 898 configurations of the $24^{3} \times 64$ ensemble with $N_{f}=2+1+1$ flavors of highly improved staggered quarks (HISQ) [53] generated by the MILC Collaboration [54]. Hypercubic smearing [55] is applied to these configurations. The lattice spacing of this ensemble is $a \approx 0.12 \mathrm{fm}$, with $M_{\pi} \approx 310 \mathrm{MeV}$. The spatial length of this ensemble is approximately $2.88 \mathrm{fm}$, which gives the $M_{\pi}^{\text {val }} L \leq 4.55$. Past finite-volume studies of nucleon LaMET quasi-PDFs [34] suggest there is a negligible likelihood of finite-volume effects in our study here; we will defer study of finite-volume systematics to future works. The nucleon two-point correlators are constructed with momentum-smeared sources [56] to obtain better signals of large-momentum results. We use momentum-smearing parameters $k= \pm 2.9$, and calculate $P_{z}=$ $n_{z} \frac{2 \pi}{L}$ with $\left|n_{z}\right| \in[0,5]$ corresponding to $0 \mathrm{GeV}$ to $2.15 \mathrm{GeV}$. We use relativistic heavy-quark (RHQ) action [57-61] with the charm mass parameter taken from Ref. [62] and treelevel tadpole-improved coefficients for the others. An advantage of using RHQ action is that as the heavy-quark mass decreases, this action goes over smoothly to the $O(a)$ improved clover actions that we use for the light and strange valence quarks.

The calculation of light nucleon $\left(M_{\pi} \approx 310 \mathrm{MeV}\right)$ twopoint correlators includes 344,064 measurements in total. We also measured 57,344 strange nucleon $\left(M_{\pi} \approx 690 \mathrm{MeV}\right)$ two-point correlators to check the pion-mass dependence. At each boost momentum, the nucleon energy is obtained through a two-state fit to the two-point correlator, $C_{2 \mathrm{pt}}(t)=\left|\mathcal{A}_{0}\right|^{2} e^{-E_{0} t}+\left|\mathcal{A}_{1}\right|^{2} e^{-E_{1} t}+\cdots$, where $E_{i}$ and $A_{i}$ are the energy and overlap factor between the lattice nucleon operator and desired state $|i\rangle$, and $i=0(i=i)$ stands for the ground (excited) state.

\footnotetext{
${ }^{2}$ While this paper was in the review process, another work involving "disconnected contributions to the proton helicity PDF" was presented by the ETMC Collaboration [51].
} 


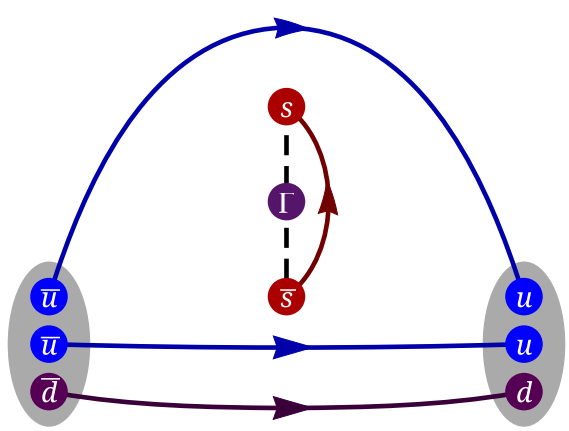

FIG. 1. Illustration of the three-point correlator involving a strange-quark long-link operator, which forms a disconnected diagram. The dashed line indicates the spatial displacement of the Wilson link with the choice of operator $\Gamma$. The gray blobs show the nucleon source and sink, separated by $t_{\text {sep }}$ in Euclidean time direction. Sea-quark and gluon interactions, although present in the lattice configurations, are omitted from this schematic diagram.

On the lattice, the only contribution to the strange quasiPDF matrix elements comes from disconnected quark loops (as shown in Fig. 1), calculated as

$$
C_{\Gamma}^{\text {loop }}=\sum_{n} \operatorname{Tr}\left[\left(S_{s, c}(n+z \hat{z}, n) \prod_{i=0}^{z-1} U_{z}(n+i \hat{z})\right) \Gamma\right],
$$

where $S_{s, c}$ are strange- and charm-quark propagators, $\Gamma=$ $\gamma_{t}$ gives the unpolarized quasi-PDF, and $n$ indexes over lattice sites. One of the main challenges to finding the nucleon strange and charm content is calculating the computationally expensive and statistically noisy disconnected diagrams. We calculate the disconnected diagrams using a stochastic estimator with noise sources accelerated by a combination of the truncated-solver method $[63,64]$, the hopping-parameter expansion [65,66], and the all-mode averaging technique [67]. These methods of calculating quark-line disconnected contributions have proven to be useful in extracting the up, down, and strange contributions to the nucleon tensor charges and setting an upper bound for beyond-Standard-Model that is dominated by the quark electric dipole moment $[68,69]$. For the disconnected strange-quark loop in this calculation, we have a total of $7,184,000$ low-precision (LP) measurements $\left(N_{\mathrm{LP}}=8000\right.$ for each configuration) and 143,680 high-precision (HP) measurements. For the disconnected charm quark loop, we have a total of 3,592,000 LP measurements $\left(N_{\mathrm{LP}}=4000\right.$ for each configuration) and 71,840 HP measurements. Once we obtain the strange/charm loop, we can construct the three-point correlators $\left(C_{3 \mathrm{pt}}\right)$ by combining it with the nucleon two-point correlator $\left(C_{2 \mathrm{pt}}\right)$,

$$
\begin{aligned}
C_{3 \mathrm{pt}}\left(t, t_{\mathrm{sep}}\right)= & \left\langle\left(C_{2 \mathrm{pt}}\left(t_{\mathrm{src}}, t_{\mathrm{sep}}\right)-\left\langle C_{2 \mathrm{pt}}\left(t_{\mathrm{src}}, t_{\mathrm{sep}}\right)\right\rangle\right)\right. \\
& \left.\cdot\left(C_{\gamma_{t}}^{\text {loop }}\left(t+t_{\mathrm{src}}\right)-\left\langle C_{\gamma_{t}}^{\text {loop }}\left(t+t_{\mathrm{src}}\right)\right\rangle\right)\right\rangle_{t_{\mathrm{scc}}},
\end{aligned}
$$

where $t_{\text {src }}$ and $t_{\text {sep }}$ are the source location and source-sink separation, respectively.

To obtain the ground-state nucleon strange matrix elements, we fit the three-point correlators, which are expanded in energy eigenstates as

$$
\begin{aligned}
C_{3 \mathrm{pt}}\left(t_{\mathrm{sep}}, t\right)= & \left|\mathcal{A}_{0}\right|^{2}\langle 0|O| 0\rangle e^{-E_{0} t_{\mathrm{sep}}} \\
& +\mathcal{A}_{0} \mathcal{A}_{1}^{*}\langle 0|O| 1\rangle e^{-E_{0} t} e^{-M_{1}\left(t_{\mathrm{sep}}-t\right)} \\
& +\mathcal{A}_{0}^{*} \mathcal{A}_{1}\langle 1|O| 0\rangle e^{-E_{0}\left(t_{\mathrm{sep}}-t\right)} e^{-E_{1} t} \\
& +\left|\mathcal{A}_{1}\right|^{2}\langle 1|O| 1\rangle e^{-E_{1} t_{\mathrm{sep}}}+\cdots
\end{aligned}
$$

where $\left\langle i^{\prime}|O| i\right\rangle$ indicates matrix elements for the ground state $\left(i=i^{\prime}=0\right)$ or excited states $\left(i^{\prime}=1\right)$. The ground-state matrix element $\langle 0|O| 0\rangle$ could be approximated by the ratio

$$
R\left(t_{\mathrm{sep}}, t\right)=\left\langle C_{3 \mathrm{pt}}\left(t_{\mathrm{sep}}, t\right)\right\rangle /\left\langle C_{2 \mathrm{pt}}\left(t_{\mathrm{sep}}\right)\right\rangle
$$

if the excited-state contamination in the data were small.
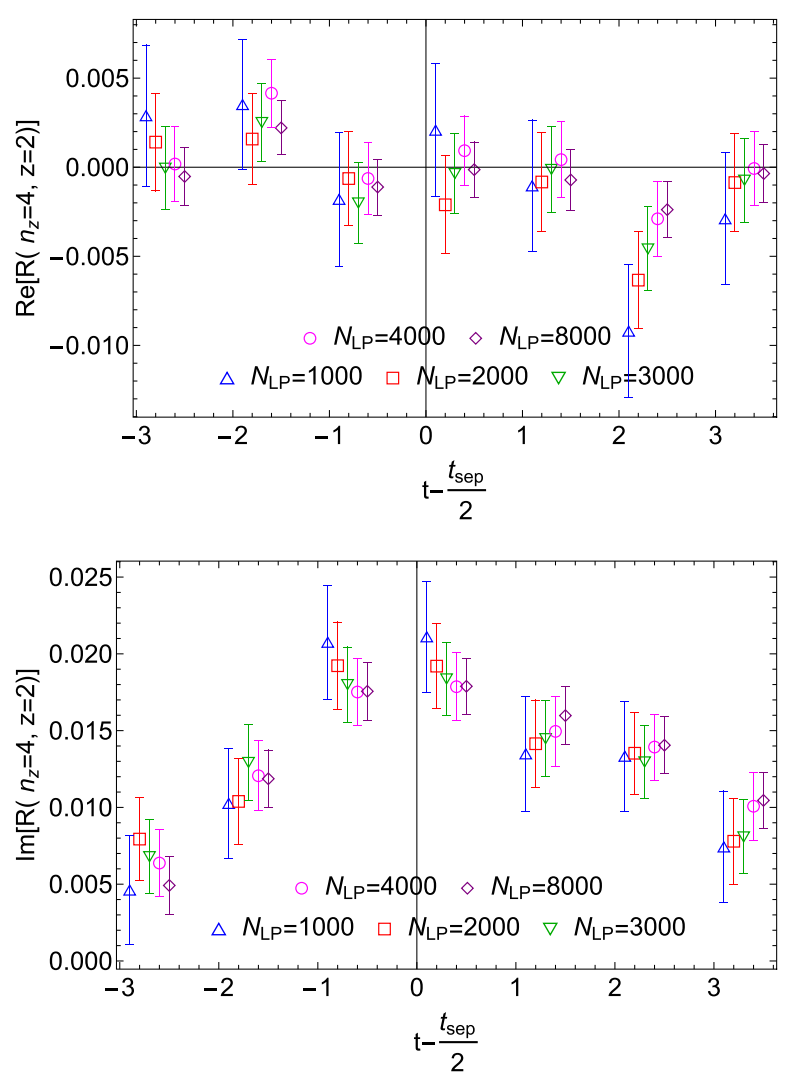

FIG. 2. Example ratio plot for real (top) and imaginary (bottom) strange matrix elements from $n_{z}=4, z=2, t_{\text {sep }}=6$ as function of insertion time $t$, centered by half of $t_{\text {sep }}$. Different data points indicate $N_{\mathrm{LP}} \in\{1000,2000,3000,4000,8000\}$ noise sources used in the calculation (slightly shifted in $t$ to make the data points visible). The error is effectively reduced by using larger $N_{\text {LP. }}$ 


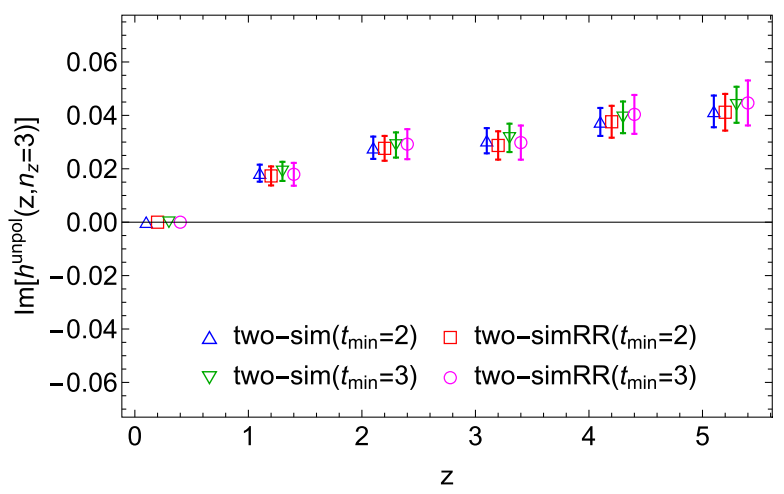

FIG. 3. Comparison of the imaginary part of the strange quasiPDF renormalized matrix elements obtained from different fit strategies at $n_{z}=3$.

Figure 2 shows one example of real and imaginary ratio plots for strange quasi-PDF operators at $n_{z}=4, z=2$ and $t_{\text {sep }}=6$ using different numbers of the low-precision sources, varying from 1000 to 8000 . We find the statistical errors consistently decrease as the $N_{\mathrm{LP}}$ increases, approximately scaling as $\frac{1}{\sqrt{N_{\mathrm{LP}}}}$.

We check the stability of the fit results using different strategies; fitting the two-point correlators of $t \in[2,10]$ to the first two states in Eq. (6), and the three-point correlators within $t_{\text {sep }} \in[6,9]$ and $t \in\left[1, t_{\text {sep }}-1\right]$ to the first three terms (two-sim) or four terms (two-simRR). An example of the fitted renormalized matrix elements is shown in Fig. 3. The fit results are consistent among different strategies. In
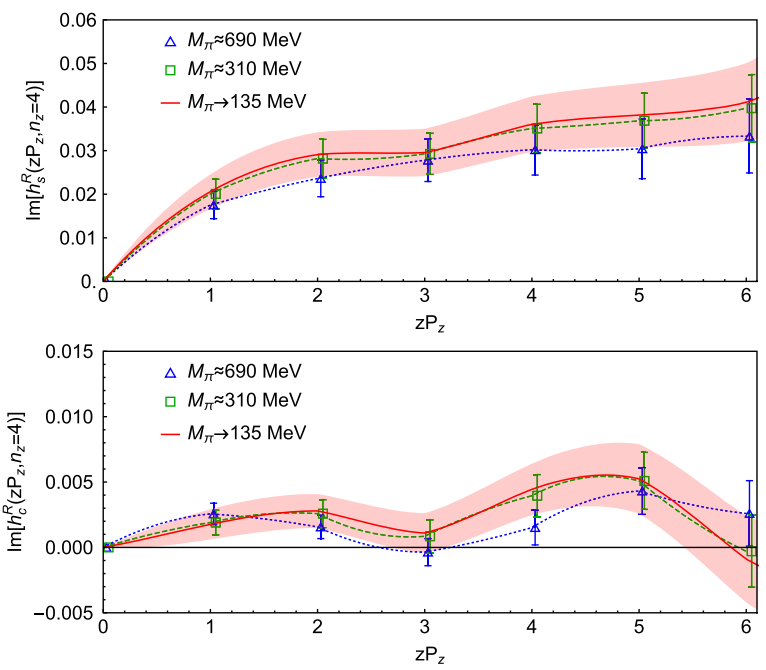

FIG. 4. Extrapolation to physical pion mass for the imaginary part of strange (top) and charm (bottom) MEs at $n_{z}=4$. The extrapolated results are very close to the $M_{\pi} \approx 310 \mathrm{MeV}$ results. The charm distribution is much smaller, thus noisier with larger relative errors. The band indicates the extrapolated matrix elements at the physical pion mass.

the remaining part of the paper, we adopt the two-sim strategy and $t_{\mathrm{min}}^{2 \mathrm{pt}}=3$ for the fits.

We then apply the renormalization factors to the bare matrix elements, in order to make comparisons with other results. We adopt NPR in the RI/MOM scheme, the same strategy as in past works [28,70], by imposing

$$
Z\left(p_{z}^{\mathrm{RI}}, \mu^{\mathrm{RI}}\right)=\left.\frac{\operatorname{Tr}\left[\mathcal{P} \sum_{s}\left\langle p, s\left|\bar{\psi}_{f}(\lambda \tilde{n}) \gamma_{t} W(\lambda \tilde{n}, 0) \psi_{f}(0)\right| p, s\right\rangle\right]}{\operatorname{Tr}\left[\mathcal{P} \sum_{s}\left\langle p, s\left|\bar{\psi}_{f}(\lambda \tilde{n}) \gamma_{t} W(\lambda \tilde{n}, 0) \psi_{f}(0)\right| p, s\right\rangle_{\text {tree }}\right]}\right|_{\substack{p^{2}=-\mu_{\mathrm{RI}}^{2} \\ p_{z}=p_{z}^{\mathrm{RI}}}}
$$

where we used the projection $\mathcal{P}=\gamma_{t}-\frac{p_{t}}{p_{x}} \gamma_{x}$. We use the NPR factors in the RI/MOM scheme calculated from Ref. [35] to obtain the renormalized matrix elements $h^{R}\left(z, p_{z}^{\mathrm{RI}}, \mu^{\mathrm{RI}}\right)=Z^{-1}\left(p_{z}^{\mathrm{RI}}, \mu^{\mathrm{RI}}\right) h(z)$ - throughout this work, we will fix the scales to $p_{z}^{\mathrm{RI}}=0, \mu^{\mathrm{RI}}=2.3 \mathrm{GeV}$.

The same quantities are also calculated with smaller statistics for the nucleon at the SU(3) point, where the lightquark masses are equal to the physical strange-quark mass, showing similar behavior with comparable error sizes. With the two mass points $M_{\pi} \approx 310 \mathrm{MeV}$ and $M_{\pi} \approx 690 \mathrm{MeV}$, we perform a naive chiral extrapolation with the form $h^{R}\left(M_{\pi}\right)=h_{\text {phys }}^{R}+c_{1}\left(M_{\pi}^{2}-M_{\pi \text {,phys }}^{2}\right)$ to estimate the matrix elements at physical pion mass $M_{\pi \text {,phys }} \approx 135 \mathrm{MeV}$. Figure 4 shows an example of chiral extrapolated results for the imaginary part of strange and charm matrix elements at boost momentum $P_{z}=1.76 \mathrm{GeV}$ as a function of $z P_{z}$, or the Ioffe time $\nu=z \cdot P$ [71] commonly used in the study of pseudo-PDFs [17]. Both extrapolated matrix elements are close to those from the $310 \mathrm{MeV}$ calculation. On the top panel, in the strange case, we observe a small pion-mass dependence between the $310 \mathrm{MeV}$ and $690 \mathrm{MeV}$ results at large $P_{z}$; the results at lighter pion mass show slightly larger distributions. On the bottom panel, we see a larger pion-mass dependence for the charm case. This extrapolation with only two masses, one of which is heavy, is just a naive estimation, but can be viewed as the matrix elements calculated at $M_{\pi}=$ $310 \mathrm{MeV}$ with systematics estimated for the heavier than physical pion mass. The charm matrix elements are smaller and show signs of oscillating statistically as a function of $z P_{z}$, whereas the strange distribution shows a tendency to keep growing as $z P_{z}$ increases.

\section{LATTICE RESULTS}

We compare our extrapolated matrix element results with those obtained from global fitting PDFs from CT18NNLO 
[72] and the NNPDF3.1NNLO [73] at $2 \mathrm{GeV}$ in $\overline{\mathrm{MS}}$ scheme provided by LHAPDF [74]; we match these to quasi-PDF matrix elements with $P_{z}=2.15 \mathrm{GeV}$ in the RI/ MOM scheme at $\mu^{\mathrm{RI}}=2.3 \mathrm{GeV}$ and $p_{z}^{\mathrm{RI}}=0$, using [33]

$$
\begin{aligned}
\tilde{h}_{s}\left(z P_{z}\right)= & \int_{-\infty}^{\infty} d x e^{i x z P_{z}} \int_{-1}^{1} \frac{d y}{|y|} C_{s / s}\left(x, y, \mu, \mu^{\mathrm{RI}}, p_{z}^{\mathrm{RI}}, P_{z}\right) s(y) \\
& +\int_{-1}^{1} \frac{d y}{|y|} C_{s / g}\left(x, y, \mu, \mu^{\mathrm{RI}}, p_{z}^{\mathrm{RI}}, P_{z}\right) g(y),
\end{aligned}
$$

with the additional term proportional to the gluon lightcone PDF being due to the strange quasi-PDF receiving a contribution at one-loop order, and from other flavors at higher-loop order. Thus, when we match the global-fit PDF to coordinate space, we use both the strange and gluon light-cone PDFs. An evaluation of the individual contributions from the strange and gluon light-cone PDFs is discussed in Appendix C. The errors of both global-fit PDFs are estimated from the Hessian PDF sets [75-77] matched to quasi-PDF matrix elements.

The real matrix elements are proportional to the integral of the difference between strange and antistrange $\left[\int d x(s(x)-\bar{s}(x)) \cos \left(x z P_{z}\right)\right]$. In our results, as shown in Fig. 5, the real quasi-PDF matrix elements are consistent with zero at $95 \%$ confidence level for most ${ }_{z} P_{z}$ points at $P_{z}>1 \mathrm{GeV}$, indicating that the strange

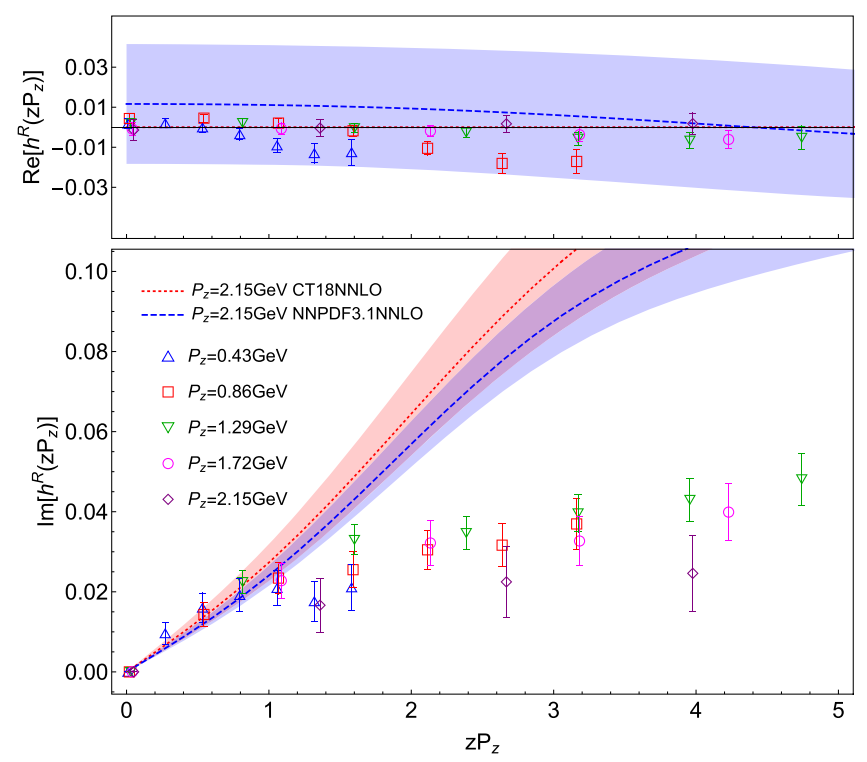

FIG. 5. The real (top) and imaginary (bottom) parts of the strange quasi-PDF matrix elements in coordinate space from our calculations at physical pion mass with $P_{z} \in[0.43,2.15] \mathrm{GeV}$, along with those from CT18 and NNPDF NNLO in RI/MOM renormalized scale of $2.3 \mathrm{GeV}$. The CT18 analysis assumes $s(x)=\bar{s}(x)$, so their results are exactly zero after matching and Fourier transformation. Our real matrix elements at $P_{z}>1 \mathrm{GeV}$ are consistent with zero, supporting strange-antistrange symmetry, while our imaginary ones are smaller than global-fit results. quark-antiquark asymmetry is likely very small. The CT18NNLO PDFs assumes a symmetric $s-\bar{s}$ distribution, so are exactly zero under the transformation with the renormalization scale we used, consistent with our findings in this work. The imaginary matrix elements are proportional to $\int d x(s(x)+\bar{s}(x)) \sin \left(x z P_{z}\right)$. We find that the matrix elements calculated at different boost momenta can have small discrepancies, but they are consistent with each other at large momentum and seem to be approaching a universal curve. The quasi-PDF matrix elements from both CT18 and NNPDF3.1 are consistent with our results within two standard deviations up to $z P_{z} \approx 2$, and deviate from our results at large $z P_{z}$, suggesting deviations at moderate to small- $x$ in the PDFs. However, although the complete matching procedure is applied to the global-fit PDF, on the lattice side only the real part of the renormalized strange quasi-PDF matrix element is complete at the RI/MOM scale $p_{z}^{\mathrm{RI}}=0$ we choose. It is not complete for the imaginary part because of mixing with the gluon operator [33]. To apply the full nonperturbative renormalization to the strange PDFs, we will need the gluon matrix elements, as well as the NPR factors for gluon and gluon-quark mixing [33]. Future studies will be necessary to discern the full strange PDF structure from lattice calculations.

Similarly, we compare the charm results with the globalfit PDFs in Fig. 6. Note that CT18 and NNPDF3.1 both assume $c(x)=\bar{c}(x)$; therefore, both of them have vanishing real matrix elements, which is consistent with the ours for the charm quasi-PDF. Our imaginary charm matrix

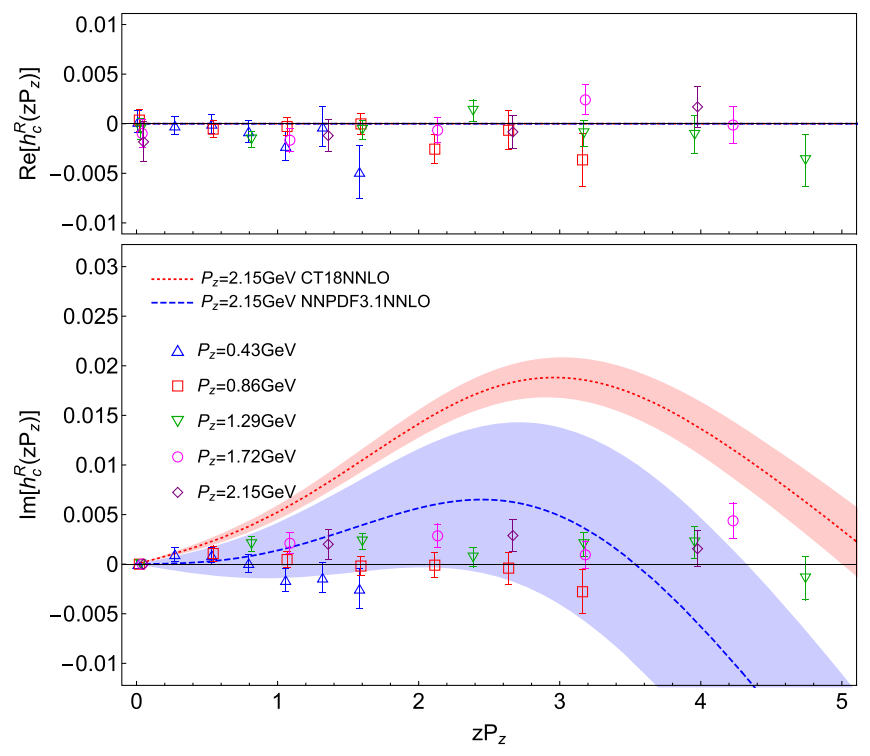

FIG. 6. The real (top) and imaginary (bottom) parts of the charm quasi-PDF matrix elements in coordinate space derived from global-fit PDFs compared with our renormalized nucleon quasi-PDF MEs at $P_{z} \in[0.43,2.15] \mathrm{GeV}$. The real part is consistent with zero, while the imaginary part is within the bounds of NNPDF3.1, but smaller than CT18 results. 
elements have much smaller magnitudes than the strange, a similar strange-charm relation also observed by global PDF fitting, such as CT18 and NNPDF3.1. The charm PDF errors from global fits are significantly different, because the CT18 charm PDF is generated by perturbatively evolving from light-quark and gluon distributions at $Q_{0}=1.3 \mathrm{GeV}$, while NNPDF3.1 numerically fitted the charm distribution. Our imaginary matrix elements are close to zero at small $z P_{z}$; at large $z P_{z}$ they are about a factor of ten smaller than the strange ones and are within the bounds of the NNPDF3.1 results. Note that the a12m310 lattice may be coarse for simulating charm. Our preliminary study with low statistics shows that the renormalized matrix elements of the same LaMET operators computed on the a $09 \mathrm{~m} 310$ lattice are consistent with $\mathrm{a} 12 \mathrm{~m} 310$ results within two sigma. (A more detailed investigation of the $\left(a m_{c}\right)$ effect will be made in future studies).

\section{CONCLUSIONS}

In this work, we made the first lattice-QCD calculations of the strange and charm parton distributions using LaMET (also called "quasi-PDF") approach on a single $(2+1+1)$-flavor HISQ ensemble with physical strange and charm masses and heavier than physical light-quark mass (resulting in a $310 \mathrm{MeV}$ pion). We found that our renormalized real matrix elements are zero within our statistical errors for both strange and charm, supporting the strange-antistrange, and charm-anticharm symmetry assumptions commonly adopted by most global PDF analyses. Our imaginary matrix elements are proportional to the sum of the quark and antiquark distribution, and we clearly see that the strange contribution is about a factor of five or larger than charm ones. They are consistently smaller than those from CT18 and NNPDF3.1, possibly due to the missing contributions from the mixing with gluon matrix elements in the renormalization. Higher statistics will be needed to better constrain the quarkantiquark asymmetry. A full analysis of lattice-QCD systematics, such as finite-volume effects and discretization, is not yet included, and plans to extend the current calculations are underway.

\section{ACKNOWLEDGMENTS}

We thank the MILC Collaboration for sharing the lattices used to perform this study. R. Z. also thanks Zhite Yu for useful discussions on the factorization. The LQCD calculations were performed using the CHROMA software suite [78] with the multigrid solver algorithm [79,80]. This research used resources of the National Energy Research Scientific Computing Center, a DOE Office of Science User Facility supported by the Office of Science of the U.S. Department of Energy under Contract No. DE-AC0205CH11231 through Energy Research Computing Allocations Process; the Extreme Science and
Engineering Discovery Environment (XSEDE), which is supported by National Science Foundation Grant No. ACI1548562; facilities of the USQCD Collaboration, which are funded by the Office of Science of the U.S. Department of Energy, Extreme Science and Engineering Discovery Environment (XSEDE), which is supported by National Science Foundation Grant No. ACI-1548562; and supported in part by Michigan State University through computational resources provided by the Institute for Cyber-Enabled Research (iCER). H. L. and R.Z. are supported by the US National Science Foundation under Grant No. PHY 1653405 "CAREER: Constraining Parton Distribution Functions for New-Physics Searches". B. Y. is supported by the Laboratory Directed Research and Development (LDRD) program of Los Alamos National Laboratory.

\section{APPENDIX A: ADDITIONAL FIGURES}

In this appendix we show some additional plots that support the conclusions of the main text.

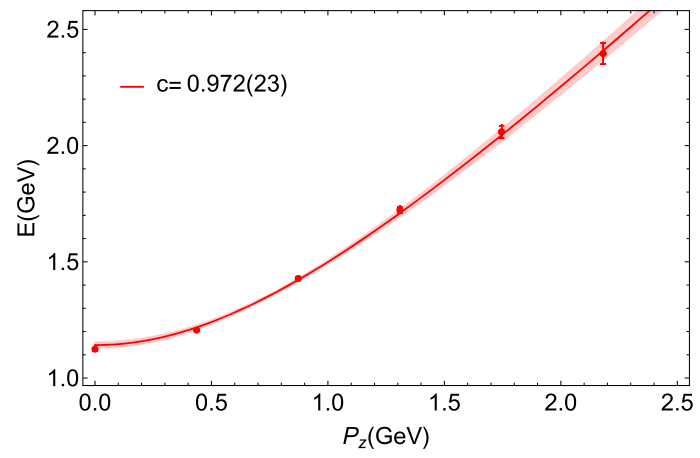

FIG. 7. The dispersion relation for the nucleon. The speed of light $c$ from the linear fit $E_{0}^{2}=c^{2} P_{z}^{2}+c^{4} M_{N}^{2}$ is slightly smaller than but consistent with 1 .

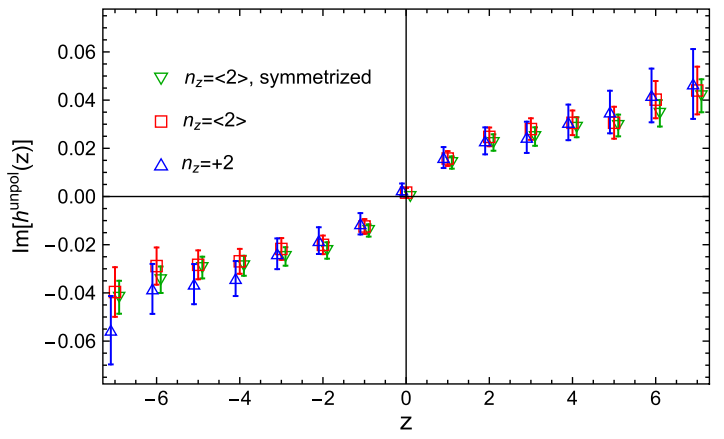

FIG. 8. Comparison of the imaginary parts of the renormalized nucleon strange-PDF matrix elements as functions of $z$ using different momentum-smearing parameters and symmetrization methods. The results are obtained at $M_{\pi} \approx 310 \mathrm{MeV}$ and nucleon boost momentum $P_{z}=0.87 \mathrm{GeV}$. 

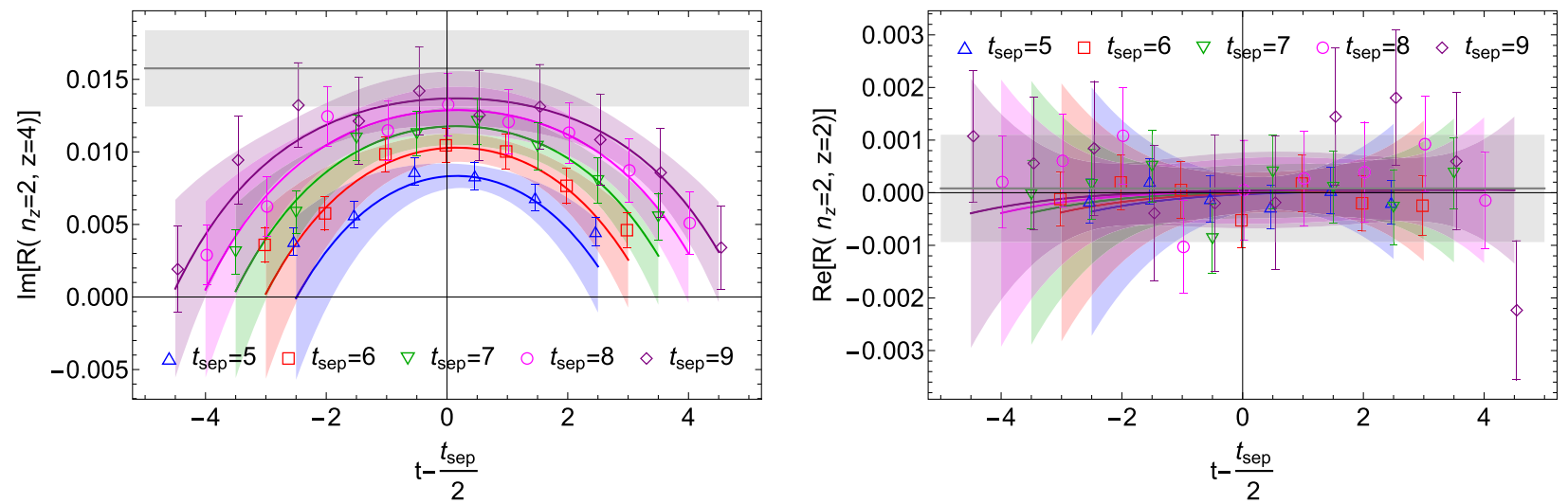

FIG. 9. Ratio plots for the imaginary part of strange quasi-PDF at $n_{z}=2, z=4$ (left) and the real part of charm quasi-PDF at $n_{z}=2$, $z=2$ (right). The ratios are plotted as data points with error bars and the fitted results are plotted in colored bands. The gray band is the ground-state matrix elements obtained from the fit.
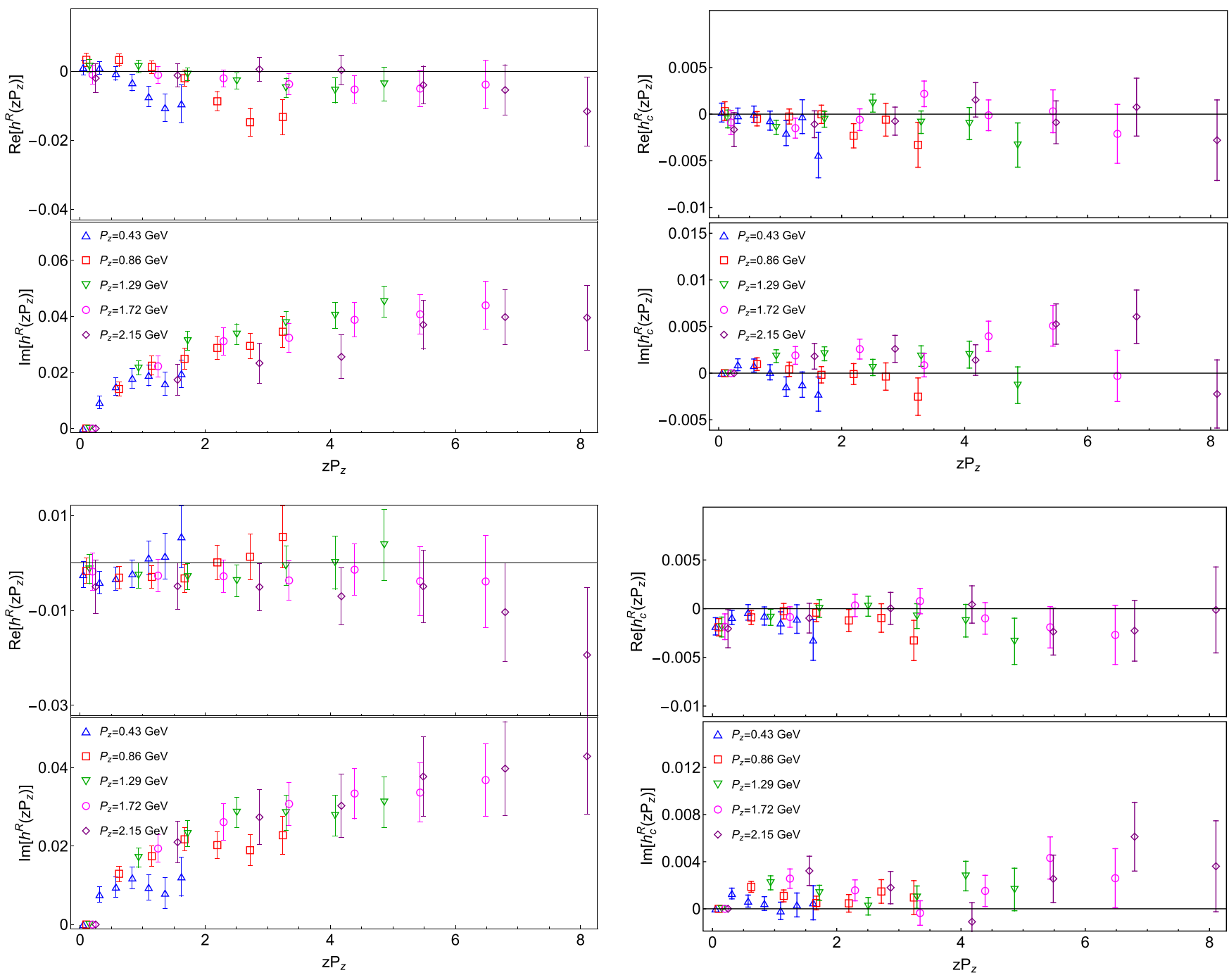

FIG. 10. RI/MOM-renormalized strange (left) and charm (right) nucleon quasi-PDF matrix element as a function of $z P_{z}$ at $P_{z} \in$ $[0.44,2.15]$ at $M_{\pi} \approx 310 \mathrm{MeV}$ (top) and $M_{\pi} \approx 690 \mathrm{MeV}$ (bottom). The real matrix elements are consistent with zero within 2 sigma for $P_{z}>1 \mathrm{GeV}$; suggesting quark-antiquark symmetry. 
Figure 7 shows the nucleon ground-state dispersion relation from our fits to the two-point correlators. We observe that the effective energy of the boosted hadrons grows slightly slower than expected, but the speed of light $c$ is consistent with 1 .

To confirm that we are observing a signal, given the small magnitude of the matrix elements, we also check whether averaging the results of the nucleon momentum in opposite directions of the smearing-momentum parameter improves the signal (which also preserves rotational symmetry in the data). Figure 8 shows example renormalized fitted imaginary matrix elements at one of the boost momenta, $P_{z}=0.88 \mathrm{GeV}$ as a function of the dimensionless parameter $z P_{z}$. It shows that the data from a single momentum versus the average of two opposite momentumsmearing results are consistent within statistical errors. We also find that averaging over opposite directions effectively increases the statistics by a factor of around two. Furthermore, to satisfy the requirement that the quasiPDF is real in momentum space, the matrix elements in coordinate space must satisfy $h(z)=h(-z)^{*}$; this is also observed in our data.

We can then utilize this symmetrization to further improve the signal in our matrix elements, as shown in Fig. 8. For the main results of this paper, we only present the matrix elements that have been averaged over momentum-smearing and symmetrized across negative link lengths.

After the above symmetrization, we show the symmetrized and renormalized strange and charm quasi-PDF matrix elements for the nucleon of $M_{\pi} \approx 310 \mathrm{MeV}$ in Fig. 10. The imaginary matrix elements from strange quasi-PDFs are about one order of magnitude larger than those of charm, which is consistent with the magnitudes obtained from the global fitting of strange and charm PDFs.

For the fit of three-point correlators, we show two selected ratio plots with fit results for the imaginary part of strange quasi-PDF at $n_{z}=2, z=4$, and the real part of charm quasi-PDF at $n_{z}=2, z=2$ in Fig. 9. The ratios calculated from correlators are presented as data points with error bars, while the fitted results are plotted as colored bands. The gray band is the ground-state matrix element we extract from the fit results. We observe that the real ratios are consistent with zero, and the imaginary ratios are consistent with the fitted results.

\section{APPENDIX B: NAIVE EXTRACTION OF THE $x$-DEPENDENCE OF THE PDF}

To extract the correct $x$-dependence for the strange and charm PDFs, we need to compute both disconnected quark matrix elements and gluon matrix elements on the lattice, renormalize them with the mixing renormalization matrix, then apply the full matching in momentum space [33]. Although we only have the contribution from the disconnected quark matrix elements, we may perform a naive extraction of the $x$-dependent strange and charm PDFs from them. A direct inverse Fourier transformation from the global-fit PDF shows that in coordinate space the strange PDF matrix element does not converge until very large $z P_{z}>1000$. Moreover, the matrix elements do not decay $h \rightarrow 0$ until $z P_{z} \approx 10^{5}$. This indicates that to obtain a reliable $x$-dependence for the strange PDF, we need go to very large $z P_{z}$ in coordinate space beyond the capability of a lattice calculation. Here, we just show a direct inverse Fourier transformation of our data truncated at $z=8$, then applied the inverse matching kernel to obtain the light-cone distribution. We show the results in Fig. 11. Because the lack of larger- $z$ contributions, which we expect to be large, the distribution in the smaller- $x$ region is significantly suppressed. The momentum dependence is also significant, although we have consistent matrix elements in coordinate space, because different momenta correspond to different truncation ranges of $z P_{z}$.
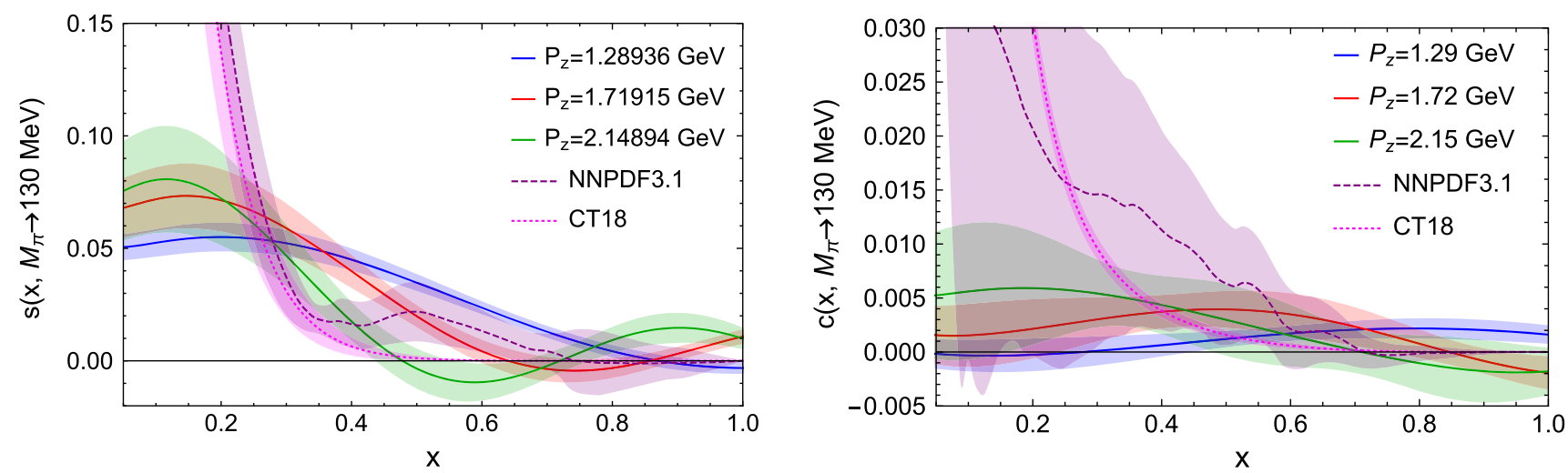

FIG. 11. The $x$-dependent strange (left) and charm (right) PDF from a naive truncated inverse Fourier transformation and inverse matching for $P_{z}=\{1.29,1.72,2.15\} \mathrm{GeV}$ at extrapolated physical pion mass. 
APPENDIX C: NONSINGLET MATCHING KERNEL FOR STRANGE AND CHARM PDFS

When $p_{z}^{\mathrm{RI}}=0$, the matching kernel in Eq. (3) satisfies

$$
C\left(x, y, \mu, \mu^{\mathrm{RI}}, p_{z}^{\mathrm{RI}}, P_{z}\right)=C\left(-x,-y, \mu, \mu^{\mathrm{RI}}, p_{z}^{\mathrm{RI}}, P_{z}\right),
$$

from which we can derive

$$
\begin{aligned}
\tilde{s}(x)+\tilde{s}(-x)= & \int_{-1}^{1} \frac{d y}{|y|} C_{q / q}\left(x, y, \mu, \mu^{\mathrm{RI}}, p_{z}^{\mathrm{RI}}, P_{z}\right) s(y)+C_{q / g}\left(x, y, \mu, \mu^{\mathrm{RI}}, p_{z}^{\mathrm{RI}}, P_{z}\right) g(y) \\
& +\int_{-1}^{1} \frac{d y}{|y|} C_{q / q}\left(-x, y, \mu, \mu^{\mathrm{RI}}, p_{z}^{\mathrm{RI}}, P_{z}\right) s(y)+C_{q / g}\left(-x, y, \mu, \mu^{\mathrm{RI}}, p_{z}^{\mathrm{RI}}, P_{z}\right) g(y) \\
= & \int_{-1}^{1} \frac{d y}{|y|} C_{q / q}\left(x, y, \mu, \mu^{\mathrm{RI}}, p_{z}^{\mathrm{RI}}, P_{z}\right) s(y)+C_{q / q}\left(-x,-y, \mu, \mu^{\mathrm{RI}}, p_{z}^{\mathrm{RI}}, P_{z}\right) s(-y) \\
& +\int_{-1}^{1} \frac{d y}{|y|} C_{q / g}\left(x, y, \mu, \mu^{\mathrm{RI}}, p_{z}^{\mathrm{RI}}, P_{z}\right) g(y)+C_{q / g}\left(-x,-y, \mu, \mu^{\mathrm{RI}}, p_{z}^{\mathrm{RI}}, P_{z}\right) g(-y) \\
& \stackrel{p_{z}^{\mathrm{RI}}=}{=} \int_{-1}^{1} \frac{d y}{|y|} C_{q / q}\left(x, y, \mu, \mu^{\mathrm{RI}}, p_{z}^{\mathrm{RI}}, P_{z}\right) s(y)+C_{q / q}\left(x, y, \mu, \mu^{\mathrm{RI}}, p_{z}^{\mathrm{RI}}, P_{z}\right) s(-y) \\
& +\int_{-1}^{1} \frac{d y}{|y|} C_{q / g}\left(x, y, \mu, \mu^{\mathrm{RI}}, p_{z}^{\mathrm{RI}}, P_{z}\right) g(y)+C_{q / g}\left(x, y, \mu, \mu^{\mathrm{RI}}, p_{z}^{\mathrm{RI}}, P_{z}\right) g(-y) \\
= & \int_{-1}^{1} \frac{d y}{|y|} C_{q / q}\left(x, y, \mu, \mu^{\mathrm{RI}}, p_{z}^{\mathrm{RI}}, P_{z}\right)(s(y)+s(-y)) \\
= & \int_{-1}^{1} \frac{d y}{|y|} C_{q / q}\left(x, y, \mu, \mu^{\mathrm{RI}}, p_{z}^{\mathrm{RI}}, P_{z}\right)(s(y)-\bar{s}(y)),
\end{aligned}
$$

where $C_{q / q}$ is the "quark-in-quark" matching kernel defined in Eq. (31) of Ref. [81], for which the leading order is just a delta function, and the one-loop order describes the difference between the quasi-PDF in RI/ MOM scheme and the light-cone PDF in $\overline{\mathrm{MS}}$ scheme. $C_{q / g}$ is the "quark-in-gluon" matching kernel defined in Eq. (74) of Ref. [33], starting from one-loop order, describing the difference between the quasi-PDF gluon-to-strange splitting in RI/MOM scheme and the light-cone gluon-tostrange splitting in $\overline{\mathrm{MS}}$ scheme. $g(x)$ is the light-cone gluon PDF with the crossing symmetry $g(-x)=-g(x)$ by convention. Note that physically the gluon PDF only has support on $x \in[0,1]$; given the antisymmetric distribution and extending the integration to $x \in[-1,1]$

$$
\int_{0}^{1} d x g(x) f(x) \rightarrow \frac{1}{2} \int_{-1}^{1} d x g(x) f(x)
$$

does not change the physics of the quasi-PDF or pseudo-PDF matching procedure. It is a convenient convention in the convolution procedure and is commonly adopted in lattice studies of the gluon PDF [33,82] because the matching kernels $C(x, y)$ are calculated for the full range $x \in[-1,1], y \in[-\infty, \infty]$. Eq. (C2) indicates that the nonsinglet matching we use here under the condition $p_{z}^{\mathrm{RI}}=0$ is applicable to the transform from $s(y)-\bar{s}(y)$ to $\tilde{s}(x)+$ $\tilde{s}(-x)$, corresponding to the real part of the quasi-PDF matrix elements.

For $s(x)+\bar{s}(x)$ component, the strange quasi-PDF $\tilde{s}$ receives both contribution $\tilde{s}_{s}$ from the matching of the strange light-cone PDF and $\tilde{s}_{g}$ from the matching of the gluon light-cone PDF at one-loop level.

$$
\begin{aligned}
\tilde{s}(x)= & \tilde{s}_{s}(x)+\tilde{s}_{g}(x) \\
= & \int_{-1}^{1} \frac{d y}{|y|} C_{q / q}\left(x, y, \mu, \mu^{\mathrm{RI}}, p_{z}^{\mathrm{RI}}, P_{z}\right) s(y) \\
& +\int_{-1}^{1} \frac{d y}{|y|} C_{q / g}\left(x, y, \mu, \mu^{\mathrm{RI}}, p_{z}^{\mathrm{RI}}, P_{z}\right) g(y),
\end{aligned}
$$

where the matching kernels $C_{q / g}$ and $C_{q / q}$ are the same as those in Eq. (C2). We use $\tilde{s}_{s}$ and $\tilde{s}_{g}$ to denote the contribution to the strange quasi-PDF from the strange light-cone PDF and gluon light-cone PDF, respectively.

To show how large the gluon contribution is, we show in Fig. 12 the full matching results of the total strange quasi$\operatorname{PDF} \tilde{s}=\tilde{s}_{s}+\tilde{s}_{g}$ (green line), and the individual contribution coming from strange $\tilde{s}_{s}$ (red line) and the gluon $\tilde{s}_{g}$ (blue line). In this example we use the CT18NNLO PDF and choose the scales to be $\mu=2 \mathrm{GeV}, \mu^{R}=2.3 \mathrm{GeV}$, 

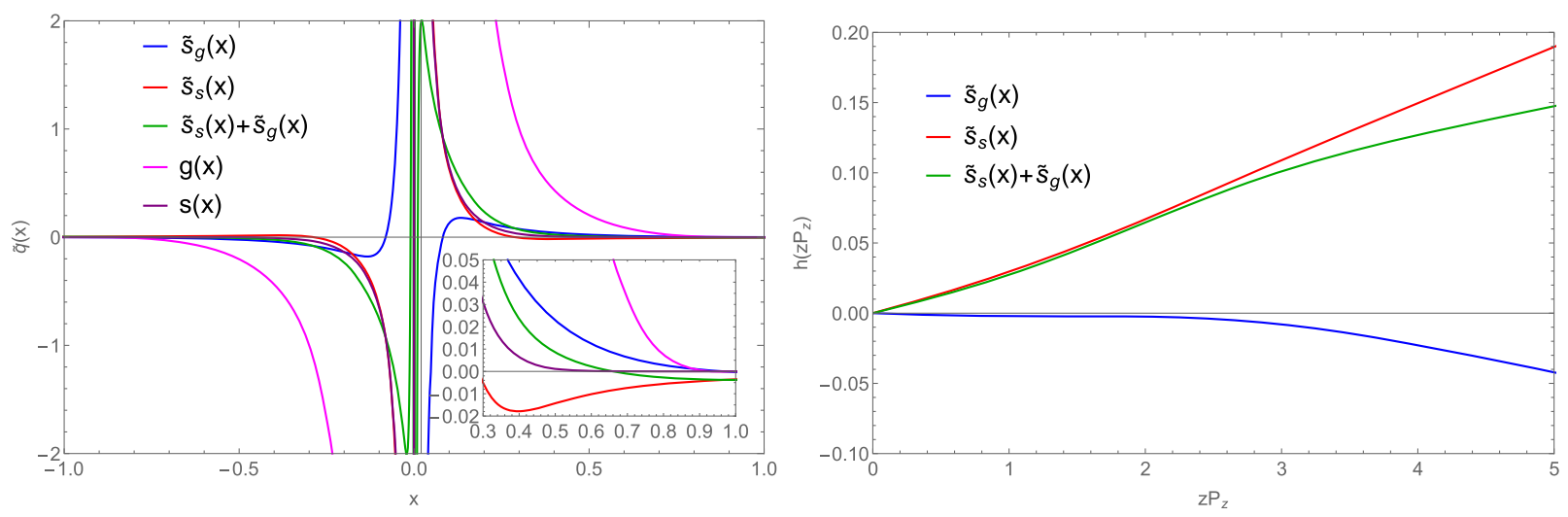

FIG. 12. Full matching results for the strange quasi-PDF in momentum space (left) and coordinate space (right) including both quark and gluon contributions. We can see that the fraction of gluon contribution (blue curve) is negative and negligible at very small $z P_{z}$, and goes up to $20 \%$ at the largest $z P_{z}$ we cover.
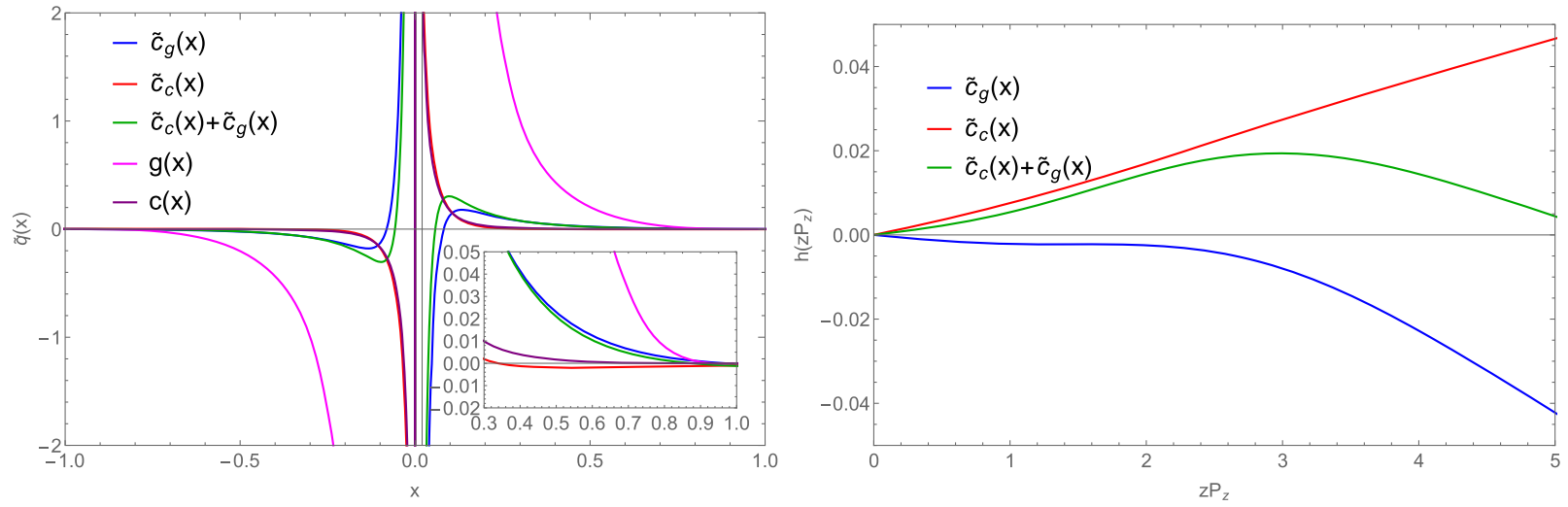

FIG. 13. Full matching results for the charm quasi-PDF in momentum space (left) and coordinate space (right) including both quark and gluon contributions. The gluon contribution (blue curve) for charm is much more significant, comparable to the charm contribution (red curve) and drag the total contribution downwards.

$p_{z}^{R}=0$, and $P_{z}=2.2 \mathrm{GeV}$. On the left-hand side of Fig. 12, we show the input light-cone strange PDF $s(x)$ (purple line) and the light-cone gluon PDF $g(x)$ (magenta line). On the right-hand side of Fig. 12, we show the corresponding matrix elements of the three strange quasiPDF results in coordinate space. It is noteworthy that the gluon contribution $\tilde{s}_{g}$ is negative at small $x$ and at almost all $z P_{z}$, and that the gluon contribution $\tilde{s}_{g}$ has smaller matrix elements compared to the strange contribution $\tilde{s}_{s}$. However, considering that the strange contribution includes the convolution with leading order $\delta\left(1-\frac{x}{y}\right)$, the gluon is still important at next-to-leading order (NLO) by compared to the gluon contribution $\tilde{s}_{g}$ as seen in the difference between strange PDF and quasi-PDF $s-\tilde{s}$. We show a similar analysis for the charm quasi-PDF in Fig. 13. In the charm case, the gluon contribution to charm $\tilde{c}_{g}=\tilde{s}_{g}$ is exactly the same as that in the strange quasi-PDF, but the charm lightcone PDF is much smaller than that of the strange. Thus, in the charm quasi-PDF the NLO gluon contribution becomes comparable to the leading-order result.
[1] S. Chatrchyan et al. (CMS Collaboration), Science 338, 1569 (2012).

[2] G. Aad et al. (ATLAS Collaboration), Science 338, 1576 (2012).
[3] G. Aad et al. (ATLAS Collaboration), J. High Energy Phys. 05 (2014) 068.

[4] S. Chatrchyan et al. (CMS Collaboration), Phys. Rev. D 90, 032004 (2014). 
[5] S. Brodsky, P. Hoyer, C. Peterson, and N. Sakai, Phys. Lett. 93B, 451 (1980).

[6] S. J. Brodsky, J. C. Collins, S. D. Ellis, J. F. Gunion, and A. H. Mueller, in 1984 DPF Summer Study on the Design and Utilization of the Superconducting Super Collider (SSC) (Snowmass 84), CO, United States (C84-06-23) (1984), p. 227.

[7] B. Harris, J. Smith, and R. Vogt, Nucl. Phys. B461, 181 (1996).

[8] M. Franz, M. V. Polyakov, and K. Goeke, Phys. Rev. D 62, 074024 (2000).

[9] S. Brodsky, A. Kusina, F. Lyonnet, I. Schienbein, H. Spiesberger, and R. Vogt, Adv. High Energy Phys. 2015, 1 (2015).

[10] T. J. Hobbs, J. T. Londergan, and W. Melnitchouk, Phys. Rev. D 89, 074008 (2014).

[11] P. Jimenez-Delgado, T. J. Hobbs, J. T. Londergan, and W. Melnitchouk, Phys. Rev. Lett. 114, 082002 (2015).

[12] X. Ji, Phys. Rev. Lett. 110, 262002 (2013).

[13] X. Ji, Sci. China Phys. Mech. Astron. 57, 1407 (2014).

[14] X. Xiong, X. Ji, J.-H. Zhang, and Y. Zhao, Phys. Rev. D 90, 014051 (2014).

[15] A. Radyushkin, Phys. Lett. B 767, 314 (2017).

[16] A. V. Radyushkin, Phys. Rev. D 96, 034025 (2017).

[17] K. Orginos, A. Radyushkin, J. Karpie, and S. Zafeiropoulos, Phys. Rev. D 96, 094503 (2017).

[18] J.-W. Chen, L. Jin, H.-W. Lin, Y.-S. Liu, Y.-B. Yang, J.-H. Zhang, and Y. Zhao, arXiv:1803.0439.

[19] H.-W. Lin, J.-W. Chen, X. Ji, L. Jin, R. Li, Y.-S. Liu, Y.-B. Yang, J.-H. Zhang, and Y. Zhao, Phys. Rev. Lett. 121, 242003 (2018).

[20] J.-H. Zhang, J.-W. Chen, L. Jin, H.-W. Lin, A. Schfer, and Y. Zhao, Phys. Rev. D 100, 034505 (2019).

[21] J.-W. Chen, H.-W. Lin, and J.-H. Zhang, arXiv:1904.12376.

[22] H.-W. Lin, J.-W. Chen, S. D. Cohen, and X. Ji, Phys. Rev. D 91, 054510 (2015).

[23] J.-W. Chen, S. D. Cohen, X. Ji, H.-W. Lin, and J.-H. Zhang, Nucl. Phys. B911, 246 (2016).

[24] H.-W. Lin, J.-W. Chen, T. Ishikawa, and J.-H. Zhang (LP3 Collaboration), Phys. Rev. D 98, 054504 (2018).

[25] C. Alexandrou, K. Cichy, V. Drach, E. Garcia-Ramos, K. Hadjiyiannakou, K. Jansen, F. Steffens, and C. Wiese, Phys. Rev. D 92, 014502 (2015).

[26] C. Alexandrou, K. Cichy, M. Constantinou, K. Hadjiyiannakou, K. Jansen, F. Steffens, and C. Wiese, Phys. Rev. D 96, 014513 (2017).

[27] C. Alexandrou, K. Cichy, M. Constantinou, K. Hadjiyiannakou, K. Jansen, H. Panagopoulos, and F. Steffens, Nucl. Phys. B923, 394 (2017).

[28] J.-W. Chen, T. Ishikawa, L. Jin, H.-W. Lin, Y.-B. Yang, J.-H. Zhang, and Y. Zhao, Phys. Rev. D 97, 014505 (2018).

[29] C. Alexandrou, K. Cichy, M. Constantinou, K. Jansen, A. Scapellato, and F. Steffens, Phys. Rev. Lett. 121, 112001 (2018).

[30] C. Alexandrou, K. Cichy, M. Constantinou, K. Jansen, A. Scapellato, and F. Steffens, Phys. Rev. D 98, 091503 (2018).

[31] Z.-Y. Fan, Y.-B. Yang, A. Anthony, H.-W. Lin, and K.-F. Liu, Phys. Rev. Lett. 121, 242001 (2018).

[32] Y.-S. Liu, J.-W. Chen, L. Jin, R. Li, H.-W. Lin, Y.-B. Yang, J.-H. Zhang, and Y. Zhao, arXiv:1810.05043.
[33] W. Wang, J.-H. Zhang, S. Zhao, and R. Zhu, Phys. Rev. D 100, 074509 (2019).

[34] H.-W. Lin and R. Zhang, Phys. Rev. D 100, 074502 (2019).

[35] H.-W. Lin, J.-W. Chen, Z. Fan, J.-H. Zhang, and R. Zhang, arXiv:2003.14128.

[36] J. Karpie, K. Orginos, A. Radyushkin, and S. Zafeiropoulos, EPJ Web Conf. 175, 06032 (2018).

[37] J. Karpie, K. Orginos, and S. Zafeiropoulos, J. High Energy Phys. 11 (2018) 178.

[38] J. Karpie, K. Orginos, A. Rothkopf, and S. Zafeiropoulos, J. High Energy Phys. 04 (2019) 057.

[39] B. Joó, J. Karpie, K. Orginos, A. Radyushkin, D. Richards, and S. Zafeiropoulos, J. High Energy Phys. 12 (2019) 081.

[40] B. Joó, J. Karpie, K. Orginos, A. V. Radyushkin, D. G. Richards, R. S. Sufian, and S. Zafeiropoulos, Phys. Rev. D 100, 114512 (2019).

[41] A. Radyushkin, Phys. Rev. D 98, 014019 (2018).

[42] J.-H. Zhang, J.-W. Chen, and C. Monahan, Phys. Rev. D 97, 074508 (2018).

[43] T. Izubuchi, X. Ji, L. Jin, I. W. Stewart, and Y. Zhao, Phys. Rev. D 98, 056004 (2018).

[44] B. Joó, J. Karpie, K. Orginos, A. V. Radyushkin, D. G. Richards, and S. Zafeiropoulos, arXiv:2004.01687.

[45] M. Bhat, K. Cichy, M. Constantinou, and A. Scapellato, arXiv:2005.02102.

[46] Y.-Q. Ma and J.-W. Qiu, Phys. Rev. Lett. 120, 022003 (2018).

[47] G. S. Bali et al., Eur. Phys. J. C 78, 217 (2018).

[48] G. S. Bali, V. M. Braun, B. Gläßle, M. Göckeler, M. Gruber, F. Hutzler, P. Korcyl, A. Schäfer, P. Wein, and J.-H. Zhang, Phys. Rev. D 98, 094507 (2018).

[49] R. S. Sufian, J. Karpie, C. Egerer, K. Orginos, J.-W. Qiu, and D. G. Richards, Phys. Rev. D 99, 074507 (2019).

[50] R. S. Sufian, C. Egerer, J. Karpie, R. G. Edwards, B. Jo, Y.-Q. Ma, K. Orginos, J.-W. Qiu, and D. G. Richards, arXiv:2001.04960.

[51] C. Alexandrou, M. Constantinou, K. Hadjiyiannakou, K. Jansen, and F. Manigrasso, Phys. Rev. Lett. 126, 102003 (2021).

[52] X. Ji, Y.-S. Liu, Y. Liu, J.-H. Zhang, and Y. Zhao, arXiv:2004.03543.

[53] E. Follana, Q. Mason, C. Davies, K. Hornbostel, G. P. Lepage, J. Shigemitsu, H. Trottier, and K. Wong (HPQCD, UKQCD Collaborations), Phys. Rev. D 75, 054502 (2007).

[54] A. Bazavov et al. (MILC Collaboration), Phys. Rev. D 87, 054505 (2013).

[55] A. Hasenfratz and F. Knechtli, Phys. Rev. D 64, 034504 (2001).

[56] G. S. Bali, B. Lang, B. U. Musch, and A. Schfer, Phys. Rev. D 93, 094515 (2016).

[57] N. H. Christ, M. Li, and H.-W. Lin, Phys. Rev. D 76, 074505 (2007).

[58] H.-W. Lin and N. Christ, Phys. Rev. D 76, 074506 (2007).

[59] A. X. El-Khadra, A. S. Kronfeld, and P. B. Mackenzie, Phys. Rev. D 55, 3933 (1997).

[60] X.-D. Ji, J.-P. Ma, and F. Yuan, Phys. Lett. B 610, 247 (2005).

[61] S. Aoki, Y. Kuramashi, and S.-I. Tominaga, Prog. Theor. Phys. 109, 383 (2003). 
[62] R. A. Briceno, H.-W. Lin, and D. R. Bolton, Phys. Rev. D 86, 094504 (2012).

[63] S. Collins, G. Bali, and A. Schafer, Proc. Sci., LATTICE2007 (2007) 141 [arXiv:0709.3217].

[64] G. S. Bali, S. Collins, and A. Schafer, Comput. Phys. Commun. 181, 1570 (2010).

[65] C. Thron, S. Dong, K. Liu, and H. Ying, Phys. Rev. D 57, 1642 (1998).

[66] C. Michael, M. Foster, and C. McNeile (UKQCD Collaboration), Nucl. Phys. B Proc. Suppl. 83, 185 (2000).

[67] T. Blum, T. Izubuchi, and E. Shintani, Phys. Rev. D 88, 094503 (2013).

[68] T. Bhattacharya, V. Cirigliano, S. Cohen, R. Gupta, A. Joseph, H.-W. Lin, and B. Yoon (PNDME Collaboration), Phys. Rev. D 92, 094511 (2015).

[69] T. Bhattacharya, V. Cirigliano, R. Gupta, H.-W. Lin, and B. Yoon, Phys. Rev. Lett. 115, 212002 (2015).

[70] I. W. Stewart and Y. Zhao, Phys. Rev. D 97, 054512 (2018).

[71] B. Ioffe, Phys. Lett. 30B, 123 (1969).

[72] T.-J. Hou et al., Phys. Rev. D 103, 014013 (2021).

[73] R. D. Ball et al. (NNPDF Collaboration), Eur. Phys. J. C 77, 663 (2017).
[74] A. Buckley, J. Ferrando, S. Lloyd, K. Nordstrm, B. Page, M. Rfenacht, M. Schnherr, and G. Watt, Eur. Phys. J. C 75, 132 (2015).

[75] J. Pumplin, D. Stump, R. Brock, D. Casey, J. Huston, J. Kalk, H. Lai, and W. Tung, Phys. Rev. D 65, 014013 (2001).

[76] J. Pumplin, D. Stump, J. Huston, H. Lai, P. M. Nadolsky, and W. Tung, J. High Energy Phys. 07 (2002) 012.

[77] S. Carrazza, S. Forte, Z. Kassabov, J. I. Latorre, and J. Rojo, Eur. Phys. J. C 75, 369 (2015).

[78] R. G. Edwards and B. Joo (SciDAC, LHPC, UKQCD Collaborations), Nucl. Phys. B, Proc. Suppl. 140, 832 (2005).

[79] R. Babich, J. Brannick, R. C. Brower, M. A. Clark, T. A. Manteuffel, S. F. McCormick, J. C. Osborn, and C. Rebbi, Phys. Rev. Lett. 105, 201602 (2010).

[80] J. C. Osborn, R. Babich, J. Brannick, R. C. Brower, M. A. Clark, S. D. Cohen, and C. Rebbi, Proc. Sci., LATTICE2010 (2010) 037.

[81] Y.-S. Liu et al. (Lattice Parton Collaboration), Phys. Rev. D 101, 034020 (2020).

[82] I. Balitsky, W. Morris, and A. Radyushkin, Phys. Lett. B 808, 135621 (2020). 